\title{
Inespecificidad persistente en amplificación isotérmica mediada por loop para diagnóstico de malaria
}

\author{
Persistent non-specificity in loop-mediated insothermal amplication for \\ malaria diagnosis
}

Laura Cristina Pachón Meza1, Sandra Milena Barrera Ayala².

\section{Resumen}

Objetivo. Describir el ensayo realizado para la estandarización de la técnica de diagnóstico para malaria, denominada amplificación isotérmica mediada por loop (LAMP). Métodos. Se extrajeron 48 muestras divididas en 22 muestras para P. falciparum, 20 de P. vivax y 6 negativas para las mismas especies. Resultados. Se observaron falsos positivos, amplificaciones específicas que evidencian la inespecificidad de la técnica. Se propone estudiar los amplicones obtenidos por medio de restricción de fragmentos y secuenciación para elucidar el origen del problema.

Palabras Clave: malaria, ampliación, isotérmica mediada por loop (LAMP), especificidad LAMP, P. falciparum, P. Vivax.

\begin{abstract}
Objetive. The present study describes the assay performed to standardize a malaria diagnostic DNA test, called loop-mediated isothermal amplification (LAMP). Methods. Extractions from 48 Plasmodium-infected blood samples (22 P. falciparum-positive samples, 20 P. vivax-positive samples and 6 negative control samples). Results. The data show false positives, unspecific amplifications that demonstrate the low specificity of the test. We propose to study the amplicons obtained by restriction fragment and sequencing to investigate the underlying cause of the problem.
\end{abstract}

Keywords: malaria, doop-mediated, isothermal, amplification (LAMP), LAMP specifity, P. falciparum, P. Vivax. 
La malaria es una enfermedad causada por parásitos del género Plasmodium spp. En humanos es producida por cinco especies, $P$. vivax, $P$. falciparum, $P$. malariae, P. ovale y $P$. knowlessi $(1,2)$. Para el 2015, fueron reportados en Colombia a través del SIVIGILA 51188 casos de malaria y en lo que va del 2016 se han documentado 47274 $(3,4)$. La Organización Mundial de la Salud (OMS) ha lanzado la Estrategia de lucha contra la malaria para los años 2016-2030, donde se hace énfasis en el diagnóstico oportuno que debe recibir todo tipo de población, fortaleciendo el sistema de vigilancia y propiciando la planificación de programas de intervención (5).

Desde el ańo 2000, cuando Notomi y colaboradores documentaron la realización de una técnica de amplificación isotérmica de ADN y le nombraron LAMP por sus siglas en inglés (Loop-mediated isothermal amplification) (6) se han reportado diversas mejoras y variaciones a ésta, como el uso de iniciadores adicionales para aumento de la eficiencia de la reacción (7), innovaciones en su forma de lectura y aplicación en la detección de agentes microbianos en diversos campos de la biotecnología (8-12) convirtiéndola en una técnica versátil apta para ser leída por más de seis metodologías adaptables al entorno de ejecución (13-22).

El objetivo del presente trabajo, realizado entre Mayo de 2015 y Junio de 2016 en el grupo de Parasitología del Instituto Nacional de Salud (INS), fue estandarizar LAMP para el diagnóstico molecular de malaria en pacientes infectados por P. vivax y $P$. falciparum.

Un total de 48 muestras provenientes de los municipios de El Bagre y Tumaco (Colombia), almacenadas en papel filtro y recolectadas en diferentes investigaciones realizadas en el INS fueron sometidas a extracción de ADN y analizadas por PCR nested y LAMP. Se incluyeron 22 positivas para monoinfección con $P$. falciparum y 20 positivas para monoinfección con $P$. vivax. También fueron incluidas 6 muestras negativas para Plasmodium $s p p$. Las muestras que participaron en el estudio, contaban con caracterización previa de gota gruesa, realizada en el grupo de Parasitología del INS, donde se identificó género y especie del parásito observado, además de la parasitemia estimada.

La cepas de $P$. falciparum usadas como estándar, corresponden a FCB-1 y 3D7 aportadas por el banco de cepas del grupo de Parasitología, perteneciente al INS. La cepa de referencia de $P$. vivax fue obtenida de la Fundación Instituto de Inmunología de Colombia.

La extracción de ADN fue realizada tomando un semicírculo de $1 \mathrm{~cm}$ de radio impregnado con sangre capilar del paciente seleccionado. Para esto, fue utilizado el QIAamp ADN Micro Kit. Siguiendo las instrucciones del fabricante, se obtuvieron eluídos de ADN purificado listos para ser sometidos a pruebas moleculares de diagnóstico. Las alícuotas de una misma extracción fueron utilizadas para realizar ambas técnicas moleculares.

La PCR nested fue ejecutada como se describió previamente (23), con ligeras modificaciones. En la primera PCR, con los iniciadores rPLU5 y rPLU6, la muestra adicionada consistió de $2 \mu \mathrm{L}$ de $\mathrm{ADN}$ purificado. $\mathrm{Al}$ igual se procedió con la $\mathrm{PCR}$ especie-específica, donde se añadieron $2 \mu \mathrm{L}$ del producto de la primera PCR, sin diluir, en lugar de uno. El producto fue visualizado en agarosa al 2\%.

El set de iniciadores utilizados en LAMP fue descrito previamente (24). La amplificación se realizó en un termociclador a una temperatura inicial de $65^{\circ} \mathrm{C}$, durante 50 minutos; posteriormente la enzima pasó a ser inactivada a $80{ }^{\circ} \mathrm{C}$ durante dos minutos. El volumen final de la reacción fue $20 \mu \mathrm{L}$, conteniendo: $1.6 \mu \mathrm{M}$ de cada uno de los iniciadores FIP y BIP, $0.2 \mu \mathrm{M}$ de F3 y B3 (c/u) y $0.8 \mu \mathrm{M}$ de Loop B y Loop F; buffer de amplificación isotérmica 1X (New England Biolabs), $0.8 \mathrm{M}$ de betaína (Sigma-Aldrich), $2.8 \mathrm{mM}$ de solución dNTPs (Sigma-Aldrich), 10 $\mathrm{mM} \mathrm{MgSO}_{4}$ (New England Biolabs), 8 unidades de Bst polimerasa 2.0 (New England Biolabs) y $1 \mu \mathrm{L}$ de reactivo de detección fluorescente FDR (Eiken Chemical Co, Ltda). 
Por cada 20 muestras procesadas, fueron incluidos cuatro controles de extracción, dos positivos y dos negativos (uno para cada especie, respectivamente). También fueron procesados cuatro controles de reacción, dos positivos (correspondientes a $\mathrm{ADN}$ cepario de cada especie) y dos negativos (agua grado molecular).

Las reacciones LAMP fueron inspeccionadas bajo luz UV en busca de fluorescencia. Para resolución de discrepancias, cualquier muestra indeterminada se comparó con los controles tanto positivos como negativos. Si la fluorescencia de los controles no era concordante con la naturaleza del resultado esperado, todas las muestras pertenecientes al lote se sometían a electroforesis en agarosa al 2\% y posterior tinción con GelRed (Biotium, Inc).

Por ser un proceso de estandarización, el ejecutor de las pruebas moleculares conocía previamente la caracterización de las muestras que fue hecha por gota gruesa, por lo que la obtención de resultados estaba guiada por los datos previos.
La amplificación isotérmica no ejecutada previa-mente en el INS, fue realizada acorde a las recomendaciones científicas actuales y siguiendo los protocolos que conservan las buenas prácticas dirigidas a técnicas moleculares (25-27). No obstante, con el montaje de la técnica empezó a observarse desde el inicio la presencia de inespecificidades y falsos positivos. Para reducir el fenómeno, a lo largo de 40 diferentes ensayos se varió la [MgSO4] utilizada, en rangos de 6 hasta $12 \mathrm{mM}$; la [enzima $B s t$ ] fue testeada en un rango de 0.02 hasta $0.32 \mathrm{U} /$ reacción (Gráficos número 1 y 2) y la [dNTPs] fue modificada desde $0.4 \mathrm{mM}$ hasta $2.8 \mathrm{mM}$. Los tiempos de reacción también fueron reducidos y se elevaron las temperaturas de amplificación a fin de reducir el apareamiento de la enzima con sectores de $\mathrm{ADN}$ inespecíficos. Todas las alternativas que se intentaron son las sugeridas en la literatura cuando se presenta este tipo de fenómenos, pero ninguna tuvo efecto $(12,25)$. Dentro de este proceso también fue cambiado el set de iniciadores utilizado, pues el primero que fue testeado había sido reportado en (28-29), pero al obtener repetidos ensayos fallidos, se cambió en su totalidad por el ya mencionado.

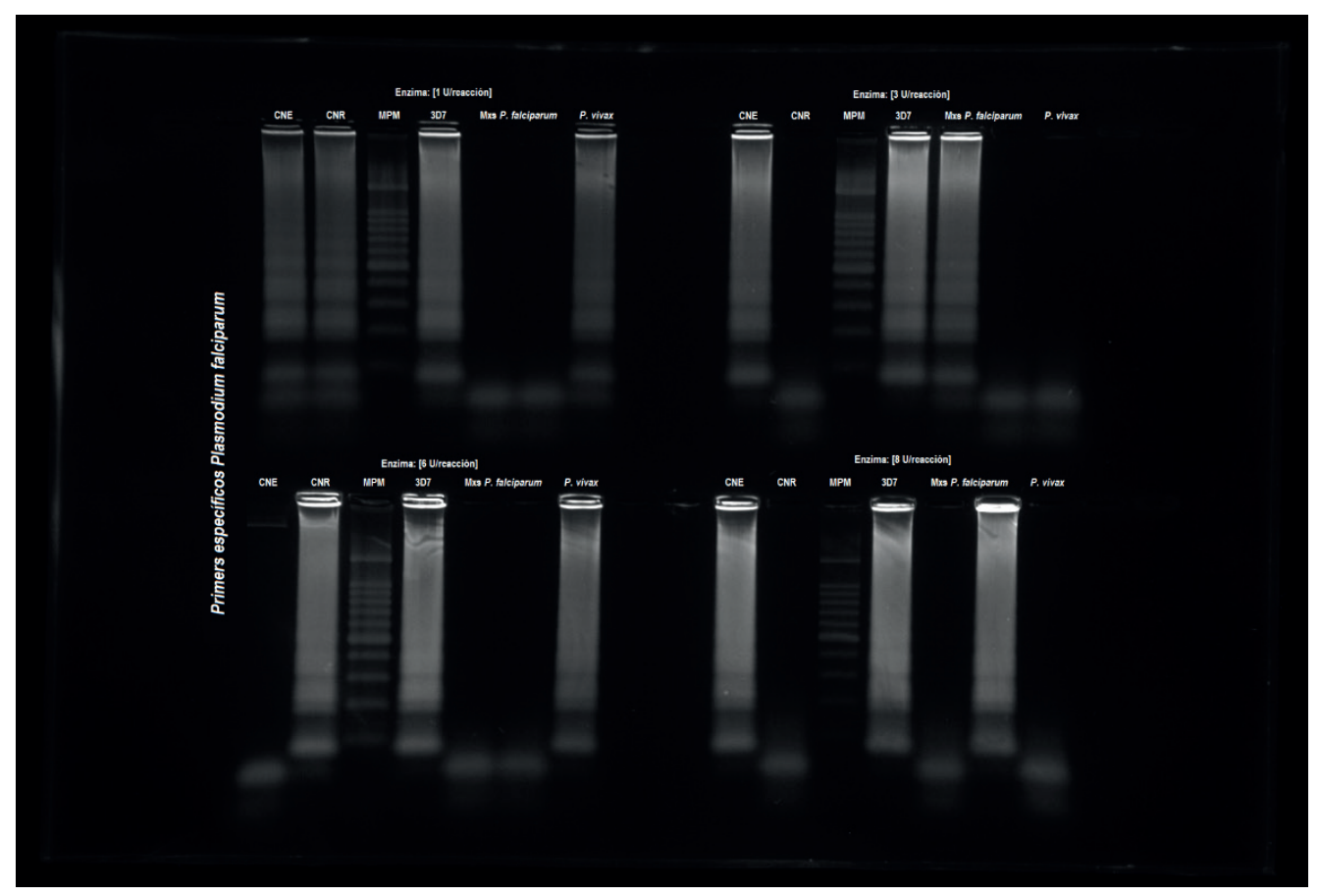

Figura 1. Electroforesis que muestra la alta inespecificidad arrojada por la reacción. Se realizó un montaje para P. falciparum disminuyendo progresivamente la concentración de la enzima con el objetivo de verificar si la presencia de amplificación inespecífica se reducía de manera proporcional. Como puede observarse, a pesar de ser montajes homólogos, los resultados no son coherentes ni replicables ni siquiera a nivel intraaensayo. 
Por el hecho de conocer la caracterización previa de las muestras, el ejecutor podía distinguir mejor entre los resultados negativos y positivos arrojados porLAMP, pero si este no fuera el caso, los resultados obtenidos eran imposibles de interpretar, llenos de pruebas indefinidas y con controles no aptos para validar el montaje.

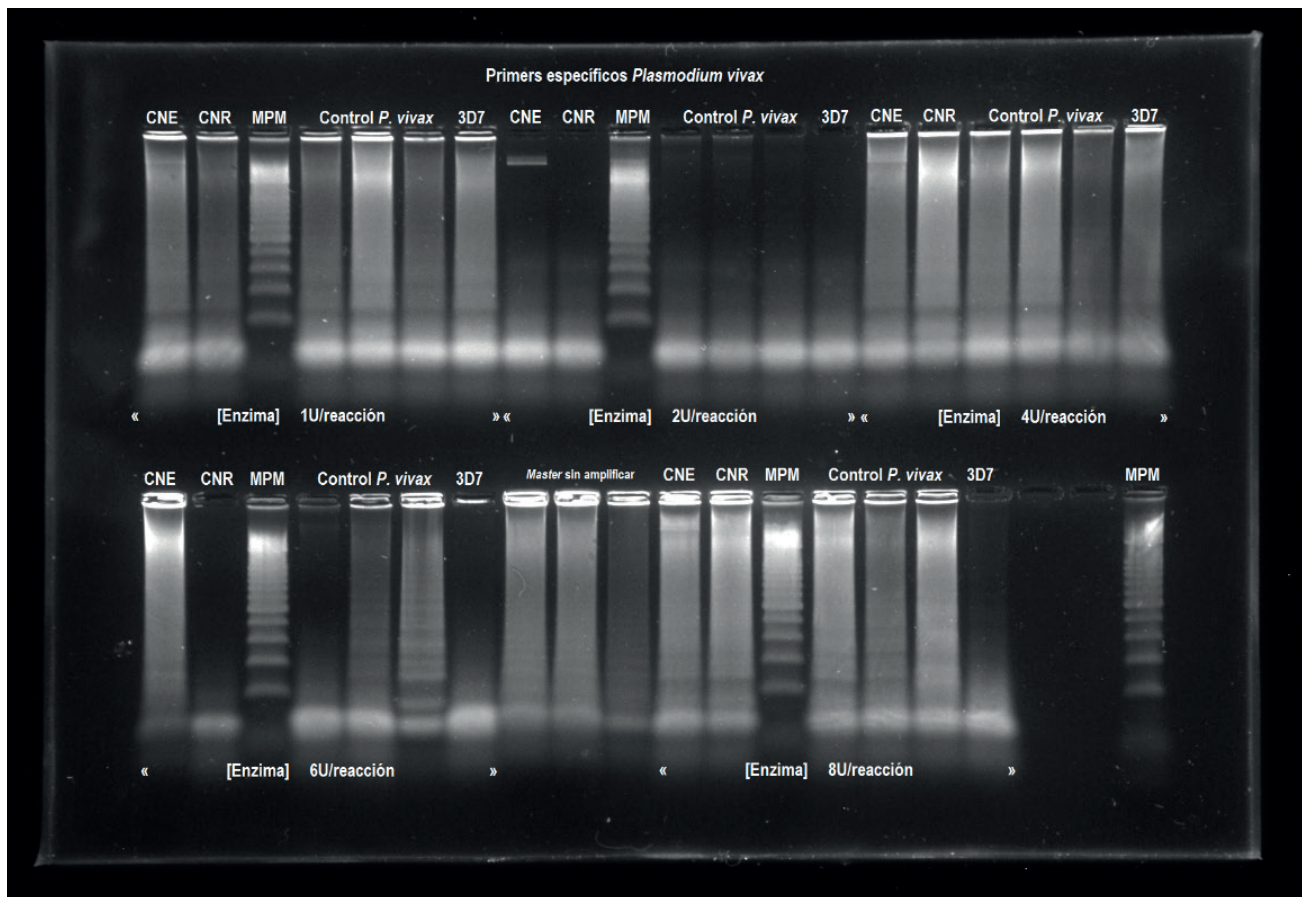

Figura 2. Electroforesis de muestras amplificadas con iniciadores $P$. vivax. Se observa que sin importar la [enzima], la amplificación inespecífica es un fenómeno recurrente. Además de esto, la especificidad arrojada es nula, pues muestras de $P$. falciparum amplifican con iniciadores no diseñados para su especie.

Los falsos negativos presentados también afectaban la lectura de la reacción, llegando así a la encrucijada entre favorecer la eficiencia de la reacción aumentando el tiempo de amplificación, exponiéndose a falsos positivos, o disminuir el tiempo de amplificación para reducir la inespecificidad, siendo propenso a que las muestras con baja cantidad inicial de ADN pasen por negativas.

La estandarización de la técnica no pudo completarse, pues a pesar de los múltiples intentos, cambios en la concentración de reactivos y alternativas puestas a prueba, la inespecificidad fue el común denominador de los ensayos, haciendo del LAMP una metodología no aplicable hasta el momento para el diagnóstico molecular de malaria en el INS. Se propone tratar el amplificado obtenido con enzimas de restricción y estudios de secuenciación, para resolver qué es lo que se está amplificando realmente y eliminar dichas interferencias permitiendo completar así el estudio.

\section{Referencias}

1. World Health Organization. World Malaria Report 2015. Geneva, Switzerland; 2015. 280 p.

2. World Health Organization. Guidelines For The Treatment of Malaria. 2015.

3. SIVIGILA. Vigilancia rutinaria por eventos departamental a semana 52 [Internet]. Bogotá; 2015. Available from: http://www. ins.gov.co/lineas-de-accion/Subdireccion-Vigilancia/sivigila/ Paginas/vigilancia-rutinaria.aspx (Agosto de 2015).

4. SIVIGILA. Vigilancia Rutinaria por evento Departamental a semana 23, 2016 [Internet]. Bogotá; 2016. Available from: http://www.ins.gov.co/lineas-de-accion/Subdireccion-Vigilancia/ sivigila/Paginas/vigilancia-rutinaria.aspx (Junio de 2016) 
5. Programa Mundial de Paludismo. Estratégica técnica mundial para o paludismo 2016-2030. Genebra,Suíça; 2016.

6. Notomi T, Okayama H, Masubuchi H, Yonekawa T, Watanabe $\mathrm{K}$, Amino N, et al. Loop-mediated isothermal amplification of DNA. Nucleic Acids Res. 2000; 28(12): E63.

7. Mori Y, Nagamine K, Tomita N, Notomi T. Detection of loopmediated isothermal amplification reaction by turbidity derived from magnesium pyrophosphate formation. Biochem Biophys Res Commun. 2001;289(1): 150-4.

8. Ferrara M, Perrone G, Gallo A, Epifani F, Visconti A., Susca A. Development of loop-mediated isothermal amplification (LAMP) assay for the rapid detection of Penicillium nordicum in dry-cured meat products. Int J Food Microbiol [Internet]. Elsevier B.V.; 2015;202:42-7. Available from: http://linkinghub.elsevier. com/retrieve/pii/S0168160515001014

9. Diribe O, North S, Sawyer J, Roberts L, Fitzpatrick N, La Ragione R. Design and application of a loop-mediated isothermal amplification assay for the rapid detection of Staphylococcus pseudintermedius. J Vet Diagn Invest [Internet]. 2014;26(1):42-8. Available from: http://www.ncbi.nlm.nih.gov/ pubmed/24398904

10. Hsieh K, Mage PL, Csordas AT, Eisenstein M, Soh HT. Simultaneous elimination of carryover contamination and detection of DNA with uracil-DNA-glycosylase-supplemented loop-mediated isothermal amplification (UDG-LAMP). Chem Commun (Camb) [Internet]. 2014;50(28):3747-9. Available from: http://www.ncbi.nlm.nih.gov/pubmed/24577617

11. Kong Q-M, Lu S-H, Tong Q-B, Lou D, Chen R, Zheng B, et al. Loop-mediated isothermal amplification (LAMP): Early detection of Toxoplasma gondii infection in mice. Parasit Vectors [Internet]. BioMed Central Ltd; 2012;5(1):2. Available from: http://www.parasitesandvectors.com/content/5/1/2

12. Senarath KD, Usgodaarachchi RB, Navaratne V, Nagahawatte a., Wijayarathna CD, Alvitigala J, et al. Non Specific Amplification with the LAMP Technique in the Diagnosis of Tuberculosis in Sri Lankan Settings. J Tuberc Res [Internet]. 2014;02(04):168-72. Available from: http://file.scirp.org/Html/3-1130069_51522. htm

13. Zhang X, Lowe SB, Gooding JJ. Brief review of monitoring methods for loop-mediated isothermal amplification (LAMP). Biosens Bioelectron [Internet]. Elsevier; 2014;61C:491-9. Available from: http://www.ncbi.nlm.nih.gov/pubmed/24949822

14. Yongkiettrakul S, Jaroenram W, Arunrut N, Chareanchim W, Pannengpetch S, Suebsing R, et al. Application of loop-mediated isothermal amplification assay combined with lateral flow dipstick for detection of Plasmodium falciparum and Plasmodium vivax. Parasitol Int [Internet]. Elsevier Ireland Ltd; 2014;63(6):777-84. Available from: http://dx.doi.org/10.1016/j.parint.2014.06.004

15. Lee P-W, Ji D-D, Liu C-T, Rampao HS, do Rosario VE, Lin I-F, et al. Application of loop-mediated isothermal amplification for malaria diagnosis during a follow-up study in São Tomé. Malar J [Internet]. 2012;11(1):408. Available from: http://www.pubmedcentral.nih.gov/articlerender. fcgi?artid $=3528453 \&$ tool $=$ pmcentrez\&rendertype $=$ abstract

16. Surabattula R, Vejandla MP, Mallepaddi PC, Faulstich K, Polavarapu R. Simple, rapid, inexpensive platform for the diagnosis of malaria by loop mediated isothermal amplification (LAMP).
Exp Parasitol [Internet]. Elsevier Inc.; 2013;134(3):333-40. Available from: http://dx.doi.org/10.1016/j.exppara.2013.03.031

17. Karthik K, Rathore R, Thomas P, Arun TR, Viswas KN, Dhama $\mathrm{K}$, et al. New closed tube loop mediated isothermal amplification assay for prevention of product cross-contamination. MethodsX [Internet]. Elsevier B.V.; 2014;1:e137-43. Available from: http:// dx.doi.org/10.1016/j.mex.2014.08.009

18. Lucchi NW, Ljolje D, Silva-Flannery L, Udhayakumar V. Use of Malachite Green-Loop Mediated Isothermal Amplification for Detection of Plasmodium spp. Parasites. PLoS One [Internet]. 2016;11(3):e0151437. Available from: http://journals.plos.org/ plosone/article?id=10.1371/journal.pone.0151437

19. Goto M, Honda E, Ogura A, Nomoto A, Hanaki KI. Colorimetric detection of loop-mediated isothermal amplification reaction by using hydroxy naphthol blue. Biotechniques. 2009;46(3):167-72.

20. Britton S, Cheng Q, Sutherland CJ, McCarthy JS. A simple, high-throughput, colourimetric, field applicable loopmediated isothermal amplification (HtLAMP) assay for malaria elimination. Malar J [Internet]. BioMed Central; 2015;14:335. Available from: http://www.pubmedcentral.nih.gov/articlerender. fcgi?artid=4552465\&tool=pmcentrez\&rendertype=abstract

21. LauY-L, FongM-Y,MahmudR, ChangP-Y,PalaeyaV,CheongF-W, et al. Specific, sensitive and rapid detection of human Plasmodium knowlesi infection by loop-mediated isothermal amplification (LAMP) in blood samples. Malar J [Internet]. 2011;10:197. Available from: http://www.pubmedcentral.nih.gov/articlerender. fcgi?artid $=3156799 \&$ tool $=$ pmcentrez $\&$ rendertype $=$ abstract

22. Lu F, Gao Q, Zhou H, Cao J, Wang W, Lim CS, et al. Molecular test for vivax malaria with loop-mediated isothermal amplification method in central China. Parasitol Res. 2012;110(6):2439-44.

23. Snounou G, Viriyakosol S, Xin Ping Zhu, Jarra W, Pinheiro L, do Rosario VE, et al. High sensitivity of detection of human malaria parasites by the use of nested polymerase chain reaction. $M o l$ Biochem Parasitol. 1993;61(2):315-20.

24. Han ET, Watanabe R, Sattabongkot J, Khuntirat B, Sirichaisinthop J, Iriko H, et al. Detection of four Plasmodium species by genusand species-specific loop-mediated isothermal amplification for clinical diagnosis. J Clin Microbiol. 2007;45(8):2521-8.

25. Tanner $\mathrm{N}$ a., Evans TC. Loop-mediated isothermal amplification for detection of nucleic acids. Curr Protoc Mol Biol. 2014;2014(January):15.14.1-15.14.14.

26. Dieffenbach CW, Dveksler GS. Setting up a PCR laboratory. Genome Res. 1993;3(2):5-14.

27. Pelt V. Ensuring PCR Quality - Laboratory Organization, PCR Optimization and Controls. In: Principles PCR. 2008.

28. Yongkiettrakul S, Jaroenram W, Arunrut N, Chareanchim W, Pannengpetch S, Suebsing R, et al. Application of loop-mediated isothermal amplification assay combined with lateral flow dipstick for detection of Plasmodium falciparum and Plasmodium vivax. Parasitol Int [Internet]. Elsevier Ireland Ltd; 2014;63(6):777-84. Available from: http://dx.doi.org/10.1016/j.parint.2014.06.004

29. Millan J, Yunda L. An Open-Access Web-based medical image atlas for collaborative Medical image sharing, processing, Web Semantic searching and analysis with uses in medical training, research and second opinion of cases. Nova. 2014;12(22):143-50 